\title{
Metabolic Engineering of Pseudomonas putida KT2440 for the Production of para-Hydroxy Benzoic Acid
}

\author{
Shiqin Yu ${ }^{1,2}$, Manuel R. Plan ${ }^{3,4}, \mathrm{Gal}$ Winter ${ }^{1,2,5}$ and Jens O. Krömer ${ }^{1,2 *}$ \\ ${ }^{1}$ Centre for Microbial Electrochemical Systems (CEMES), The University of Queensland, Brisbane, QLD, Australia, \\ ${ }^{2}$ Advanced Water Management Centre (AWMC), The University of Queensland, Brisbane, QLD, Australia, ${ }^{3}$ Australian \\ Institute for Bioengineering and Nanotechnology (AIBN), The University of Queensland, Brisbane, QLD, Australia, \\ ${ }^{4}$ Metabolomics Australia (Queensland Node), The University of Queensland, Brisbane, QLD, Australia, ${ }^{5}$ School of Science \\ and Technology, The University of New England, Armidale, NSW, Australia
}

OPEN ACCESS

Edited by:

Xuefeng Lu,

Qingdao Institute of Bioenergy and Bioprocess Technology (CAS), China

Reviewed by:

Xueli Zhang,

Tianjin Institute of Industrial Biotechnology (CAS), China

Daehee Lee,

Korea Research Institute of Bioscience and Biotechnology,

South Korea

Mingfeng Cao,

lowa State University, USA

*Correspondence:

Jens O. Krömer

j.kromer@uq.edu.au

Specialty section:

This article was submitted to

Synthetic Biology,

a section of the journal

Frontiers in Bioengineering and

Biotechnology

Received: 16 September 2016

Accepted: 10 November 2016

Published: 28 November 2016

Citation:

Yu S, Plan MR, Winter G and Krömer JO (2016) Metabolic

Engineering of Pseudomonas putida KT2440 for the Production of para-Hydroxy Benzoic Acid. Front. Bioeng. Biotechnol. 4:90. doi: 10.3389/fbioe.2016.00090
para-Hydroxy benzoic acid (PHBA) is the key component for preparing parabens, a common preservatives in food, drugs, and personal care products, as well as high-performance bioplastics such as liquid crystal polymers. Pseudomonas putida KT2440 was engineered to produce PHBA from glucose via the shikimate pathway intermediate chorismate. To obtain the PHBA production strain, chorismate lyase UbiC from Escherichia coli and a feedback resistant 3-deoxy-D-arabino-heptulosonate-7-phosphate synthase encoded by gene aroG ${ }^{\mathrm{D} 14 \mathrm{~N}}$ were overexpressed individually and simultaneously. In addition, genes related to product degradation (pobA) or competing for the precursor chorismate ( $p h e A$ and trpE) were deleted from the genome. To further improve PHBA production, the glucose metabolism repressor hex $R$ was knocked out in order to increase erythrose 4-phosphate and NADPH supply. The best strain achieved a maximum titer of $1.73 \mathrm{~g} \mathrm{~L}^{-1}$ and a carbon yield of $18.1 \%$ (C-mol C-mol-1) in a non-optimized fed-batch fermentation. This is to date the highest PHBA concentration produced by $P$. putida using a chorismate lyase.

Keywords: Pseudomonas putida KT2440, para-hydroxy benzoic acid, shikimate pathway, metabolic engineering

\section{INTRODUCTION}

The aromatic hydroxy acid, para-Hydroxy benzoic acid (PHBA) is commonly used in the chemical industry. It is a key component in the manufacturing of liquid crystal polymers (LCP) with highvalue applications in the thermoplastics market (Ibeh, 2011). PHBA is also used to prepare paraben preservatives in the cosmetics, food, and pharmaceutical industries. Current production of PHBA is based on petroleum-derived chemicals through the Kolbe-Schmitt reaction. However, the reaction's requirements for high-temperature/high-pressure conditions, along with the problem of by-product formation (Yoshida and Nagasawa, 2007), make the production of this aromatic compound relatively expensive. The volatility of raw material prices and decreasing availability in the future, together with environmental concerns are driving the development of a renewable and sustainable process for PHBA production (Erickson et al., 2012; Gavrilescu, 2014).

Microbial production of chemicals from renewable resources is a sustainable alternative and the bio-production pathways for aromatics and aromatic derivatives have been intensively studied for 
the past decades (Wierckx et al., 2005; Verhoef et al., 2007; Weber et al., 2012; Krömer et al., 2013; Lin et al., 2014; Averesch et al., 2016). The shikimate pathway is the key pathway for the synthesis of the aromatic amino acids L-tryptophan (Trp), L-phenylalanine
(Phe), and L-tyrosine (Tyr), the synthesis of quinones, folates, secondary metabolites, and derived compounds including many commercially valuable compounds (Figure 1) (Gosset, 2009; Karpf and Trussardi, 2009; Koma et al., 2012; Curran et al.,

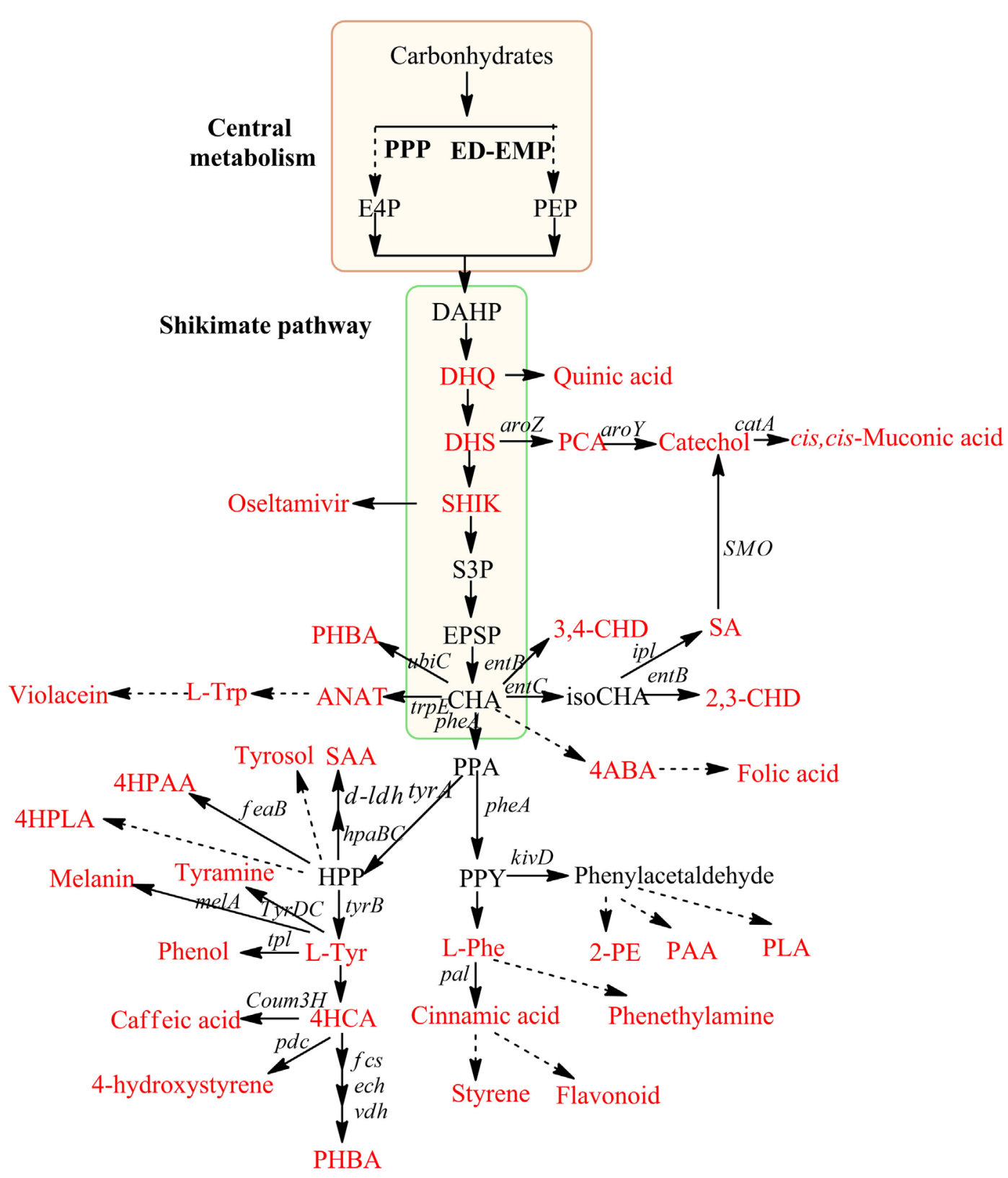

FIGURE 1 | Production of aromatic compounds via shikimate pathway in microbes. Red framed box indicates central metabolism, and green framed box indicates shikimate pathway. Compounds have industrial interest are highlighted in red color. Dashed arrows indicate multi-enzymatic synthesis. DHQ, 3-dehydroquinate; DHS, 3-dehydroshikimate; SHIK, shikimate; S3P, shikimate-3-phosphate; EPSP, 5-enolpyruvyl-shikimate 3-phosphate; CHA, chorismate; HPP, 4-hydroxy phenyl pyruvate; ANAT, anthranilate; PPA, prephenate; PPY, phenyl pyruvate; 4ABA, 4-aminobenzoic acid; SAA, salvianic acid A; 4HCA, p-hydroxy cinnamic acids; PLA, phenyl lactic acid; 4HPLA, 4-hydroxy phenyl lactic acid; 2-PE, phenyl ethanol; PAA, phenyl acetic acid; 4HPAA, 4-hydroxy phenyl acetic acid; 2,3-CHD, S,S-2,3-dihydroxy-2,3-dihydrobenzoic acid; 3,4-CHD S,S-3,4-dihydroxy-3,4-dihydrobenzoic acid; aroZ, 3-DHS dehydratase; aroY, PCA decarboxylase; catA, catechol 1,2-dioxygenase; feaB, phenylacetaldehyde dehydrogenase; tpl, Tyr phenol lyase from Pantoea agglomerans; tyrDC, tyramine decarboxylase gene from Lactobacillus brevis; pdc, $4 \mathrm{HCA}$ decarboxylase from L. plantarum; tyrB, tyrosine aminotransferase; hpaBC, an endogenous hydroxylase from E. coli; Idh, lactate dehydrogenase from L. pentosus; pal, Phe-ammonia lyase/Tyr-ammonia lyase from Rhodosporidium toruloides; kivD, phenylpyruvate decarboxylase tyrA, biofunctional chorismate mutase/prephenate dehydrogenase; entB, isochorismatase; entC, isochorismate synthase; melA, tyrosinase; fcs, $p$-coumaroyl-CoA synthetase; ech, $p$-coumaroyl-CoA hydratase/lyase; $v d h, p$-hydroxybenzaldehyde dehydrogenase; ipl, isochorismate pyruvate lyase; SMO, salicylate 1-monoxygenase; pheA, bifunctional chorismate mutase/prephenate dehydratase; tal, tyrosine ammonia lyase; Coum3H, 4-coumarate hydroxylase; ubic, chorismate. 
2013; Zhang and Stephanopoulos, 2013). The pathway links carbohydrate metabolism to aromatic compound biosynthesis by converting phosphoenolpyruvate (PEP) and D-erythrose 4-phosphate (E4P) from the central carbon metabolism into 3-deoxy-D-arabino-heptulosonate-7-phosphate (DAHP), after a sequence of seven reactions chorismate, a universal precursor for aromatic amino acids and other aromatic compounds, is formed (Bentley, 1990). Microbial production of PHBA has been studied in engineered strains of Escherichia coli (Barker and Frost, 2001), Klebsiella pneumoniae (Müller et al., 1995), and in Saccharomyces cerevisiae (Krömer et al., 2013; Williams et al., 2015). Barker and colleagues further engineered $E$. coli L-tryptophan-producing strain D2704 by inserting extra copies of genes from shikimate pathway, aroA (5-enolpyruvylshikimate 3-phosphate synthase), aroL (shikimate kinase), aroB (3-dehydroquinate synthase), and $\operatorname{aroF}$ (feedback-insensitive DAHP synthase) as well as overexpressed $u b i C$ (chorismate lyase) and $t k t A$ (transketolase), achieved a titer of $12 \mathrm{~g} \mathrm{~L}^{-1}$ and a C-mole yield of $13 \%$. Further increase of the yield was unattainable due to the toxic effect this concentration of PHBA has on E. coli. Additionally, a significant amount by-product formation was observed in the fermentation and the introduction of many unbalanced genes may lead to metabolic burdens resulting in an undesired fermentation performance.

Pseudomonas putida is well known for its versatile metabolism, fast growth rate with simple nutrient requirement, high robustness against harsh environmental conditions, and low cellular maintenance demand. Moreover, $P$. putida has a natural high resistance to PHBA. Few or no by-product formation such as acetate, glycerol, or ethanol which are frequently observed in industrial production host E. coli, Bacillus subtilis, and S. cerevisiae (Ebert et al., 2011; Poblete-Castro et al., 2012). Furthermore, P. putida almost exclusively use Entner-Doudoroff pathway (ED pathway) enabling it generates more NADPH which is important for maximization product titer ( $\mathrm{Ng}$ et al., 2015) and prevention oxidative stress (Chavarría et al., 2013). The production of shikimate pathway-derived chemicals will benefit from higher NADPH regeneration rate as the conversion of 3-dehydroshikimate into shikimate needs NADPH as the cofactor. All these features not only endow it as a flexible cell factory for bulk chemicals production but also highlight this strain as a promising workhorse for toxic chemicals production. A number of examples demonstrate its great potential in the production of a wide range chemicals, especially toxic aromatics such as phenol (Wierckx et al., 2005), PHBA (Verhoef et al., 2007), 4-hydroxy styrene (Verhoef et al., 2009), 3-nitrocatechol (Prakash et al., 2010), and 4-hydroxy quinaldine (Ütkür et al., 2011). But it is also a suitable host for the production of more traditional bio-products such as poly-hydroxy alkanoates (Le Meur et al., 2012).

Metabolic engineering of $P$. putida for the production of PHBA was first described in strain S12palB1, this strain was able to produce a maximum titer of $12.9 \mathrm{mM}$ PHBA with a C-mole yield of $8.5 \%$ on glycerol in a carbon-limited fed-batch fermentation (Verhoef et al., 2007). Yields were further increased by a mixed-substrate feeding strategy (Meijnen et al., 2011). This PHBA production strain was metabolically engineered based on the coumarate pathway (tyrosine degradation, Figure 1), which has a lower theoretical yield than production of PHBA using the chorismate lyase (UbiC) (Krömer et al., 2013). In addition, further improvement of this strain was difficult as the platform strain was based on random nitrosoguanidine (NTG) mutagenesis, and the unknown genetic basis limits strain improvement.

Here, we present the production of PHBA from glucose using the more efficient chorismate lyase route in P. putida KT2440 using rational engineering. Increased PHBA production was achieved through genetic deletion of PHBA degrading pathways as well as pathways competing for chorismate. In addition to overexpression of $u b i C$, a feedback-resistant DAHP synthase encoded by aro $G^{D 146 N}$ was also overexpressed. Finally, we explored the effect of deletion of the gene encoding for glucose metabolism repressor hexR and its effect on PHBA production.

\section{MATERIALS AND METHODS}

\section{Bacterial Strains and Plasmids}

Bacterial strains and plasmids used in this study are listed in Table 1. P. putida KT2440 (DSMZ 6125) was used as production host, and E. coli XL10-Gold (Agilent Technologies Inc.) was used to prepare all plasmids except the R6K plasmids which were prepared in E. coli DH5 $\alpha$ p pir (Martinez-Garcia and de Lorenzo, 2011).

\section{Chemicals and Reagents}

All chemicals are of analytical purity, purchased from SigmaAldrich (Australia) or Chem-Supply (Australia). Yeast extract and tryptone were purchased from Merck and Oxoid (Australia). Restriction enzymes, T4 ligase, and DNA polymerase were purchased from New England Biolabs (Australia) and were used according to the supplier's recommendation. Plasmid isolations or DNA fragment purifications were done using GeneJET kits for Plasmid Miniprep, Gel Extraction, or the PCR Purification (Thermo Fisher Scientific Australia Pty. Ltd.).

\section{Media}

Lysogeny broth (LB) consisted of $10 \mathrm{~g} \mathrm{~L}^{-1}$ tryptone, $5 \mathrm{~g} \mathrm{~L}^{-1}$ yeast extract, and $5 \mathrm{~g} \mathrm{~L}^{-1}$ sodium chloride (Lennox, 1955) and was used to culture E. coli and P. putida strains. Antibiotics of the final concentration of $50 \mu \mathrm{g} \mathrm{mL} \mathrm{m}^{-1}$ kanamycin or $50 \mu \mathrm{g} \mathrm{mL} \mathrm{m}^{-1}$ gentamycin were added into medium when it was needed. To make solid plates, $1.5-1.7 \%(\mathrm{w} / \mathrm{v})$ agar was added into the medium.

Fully chemically defined media were used in shake flask and bioreactor experiments with the following composition: chemically defined medium (CDM) for flask cultivation contained per liter: $6 \mathrm{~g} \mathrm{Na}_{2} \mathrm{HPO}_{4}, 3 \mathrm{~g} \mathrm{KH}_{2} \mathrm{PO}_{4}, 2 \mathrm{~g} \mathrm{NH}_{4} \mathrm{Cl}$, and $0.2 \mathrm{~g} \mathrm{MgSO}_{4} \cdot 7 \mathrm{H}_{2} \mathrm{O}$ and $15 \mathrm{mg} \mathrm{CaCl}{ }_{2} \cdot 2 \mathrm{H}_{2} \mathrm{O}$, and $1 \mathrm{~mL}$ trace element solution $\left(1.5 \mathrm{~g} \mathrm{~L}^{-1}\right.$ $\mathrm{FeCl}_{3} \cdot 6 \mathrm{H}_{2} \mathrm{O}, 0.15 \mathrm{~g} \mathrm{~L}^{-1} \mathrm{H}_{3} \mathrm{BO}_{3}, 0.03 \mathrm{~g} \mathrm{~L}^{-1} \mathrm{CuSO}_{4} \cdot 5 \mathrm{H}_{2} \mathrm{O}, 0.18 \mathrm{~g} \mathrm{~L}^{-1}$ $\mathrm{KI}, 0.12 \mathrm{~g} \mathrm{~L}^{-1} \mathrm{MnCl}_{2} \cdot 4 \mathrm{H}_{2} \mathrm{O}, 0.06 \mathrm{~g} \mathrm{~L}^{-1} \mathrm{Na}_{2} \mathrm{MoO}_{4} \cdot 2 \mathrm{H}_{2} \mathrm{O}, 0.023 \mathrm{~g} \mathrm{~L}^{-1}$ $\mathrm{NiCl}_{2} \cdot 6 \mathrm{H}_{2} \mathrm{O}, 0.12 \mathrm{~g} \mathrm{~L}^{-1} \mathrm{ZnSO}_{4} \cdot 7 \mathrm{H}_{2} \mathrm{O}, 0.15 \mathrm{~g} \mathrm{~L}^{-1} \mathrm{CoCl}_{2} \cdot 6 \mathrm{H}_{2} \mathrm{O}$, and $10 \mathrm{~g} \mathrm{~L}^{-1}$ EDTA). This medium was supplemented with $5 \mathrm{~g} \mathrm{~L}^{-1}$ glucose as sole carbon source, $50 \mu \mathrm{g} \mathrm{mL}^{-1}$ kanamycin (where appropriate), $0.5 \mathrm{mmol} \mathrm{L}^{-1} \operatorname{Trp}$, and $0.5 \mathrm{mmol} \mathrm{L}^{-1}$ phenyl pyruvate (PPY). 


\begin{tabular}{|c|c|c|}
\hline & Characteristics & Reference \\
\hline \multicolumn{3}{|l|}{ Plasmids } \\
\hline pSEVA234 & & Silva-Rocha et al. (2013) \\
\hline pSEVA234-ubiC & $\mathrm{km}^{\mathrm{R}}$, expression vector containing the gene of ubiC & This study \\
\hline pSEVA234-UA & $\mathrm{km}^{\mathrm{R}}$, expression vector containing the gene of $u$ biC and the gene aroG ${ }^{D 146 N}$ mutant DAHP synthase & This study \\
\hline pEMG & & Martinez-Garcia and de Lorenzo (2011) \\
\hline pEMG-pobATS1TS2 & $\mathrm{km}^{\mathrm{R}}$, using for pobA gene deletion from genome & This study \\
\hline pEMG-pheATS1TS2 & $\mathrm{km}^{\mathrm{R}}$, using for pheA gene deletion from genome & This study \\
\hline pEMG-trpETS1TS2 & $\mathrm{km}^{\mathrm{R}}$, using for trpE gene deletion from genome & This study \\
\hline pEMG-hexRTS1TS2 & $\mathrm{km}^{\mathrm{R}}$, using for hexR gene deletion from genome & This study \\
\hline \multicolumn{3}{|l|}{ Stains' name } \\
\hline E. coli XL10-Gold & $\begin{array}{l}\text { endA1 glnV44 recA1 thi-1 gyrA96 relA1 lac Hte } \Delta \text { (mcrA)183 } \Delta \text { (mcrCB-hsdSMR-mrr)173 tet }{ }^{R} \\
F^{\prime}\left[\text { proAB laclaZ } \triangle M 15 \text { Tn10 }\left(\text { Tet }^{R} \text { Amy } \mathrm{Cm}^{R}\right)\right]\end{array}$ & Stratagene/agilent technologies \\
\hline E. coli DH5 $\alpha \lambda$ pir & $\begin{array}{l}\text { endA1 hsdR17 glnV44 (=supE44) thi-1 recA1 gyrA96 relA1 } \varphi 80 d l a c \Delta(l a c Z) M 15 \Delta(l a c Z Y A-a r g F) \cup 169 \\
\text { zdg-232::Tn10 uidA::pir+ }\end{array}$ & Martinez-Garcia and de Lorenzo (2011) \\
\hline SO & Pseudomonas putida KT2440 DSM 6125, wild type & DSMZ \\
\hline S1 & The stain SO knockout the gene pobA and overexpress gene $u b i C$ & This study \\
\hline S2 & $\mathrm{S} 2$ derived from strain $\mathrm{S} 1$ by the further deletion of pheA, overexpress gene ubiC & This study \\
\hline S3 & S3 derived from strain S2 by the further deletion of trpE, overexpress gene ubiC & This study \\
\hline S4 & S4 derived from strain S3 by the further deletion of hexR, overexpress gene ubiC & This study \\
\hline S5 & S5 derived from strain S3 by the overexpression of $u b i C$ and aroG ${ }^{D 146 N}$ & This study \\
\hline S6 & S6 derived from strain $\mathrm{S} 4$ by the overexpression of $u b i C$ and aroG ${ }^{D 146 N}$ & This study \\
\hline
\end{tabular}

$K m^{R}$, kanamycin resistance.

Based on Sun's protocol two different media were used for the seed culture and the fermentation in bioreactor experiments (Sun et al., 2006). Inoculum was prepared in seed medium containing the following components per liter:

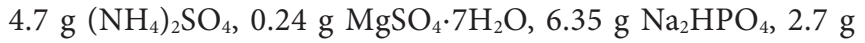
$\mathrm{KH}_{2} \mathrm{PO}_{4}, 9.0 \mathrm{~g}$ glucose, $15 \mathrm{mg} \mathrm{CaCl}_{2} \cdot 2 \mathrm{H}_{2} \mathrm{O}, 0.75 \mathrm{~g}$ tryptone, $0.3 \mathrm{~g} \mathrm{NaCl}$ and $0.15 \mathrm{~g}$ yeast extract, $1 \mathrm{~mL}$ trace metal solution, $0.5 \mathrm{mmol}$ Trp and $1 \mathrm{mmol} \mathrm{PPY}$, and $50 \mathrm{mg}^{-1}$ kanamycin. CDM was modified for bioreactor initial fermentation containing per liter: $4.7 \mathrm{~g}\left(\mathrm{NH}_{4}\right)_{2} \mathrm{SO}_{4}, 0.24 \mathrm{~g} \mathrm{MgSO}_{4} \cdot 7 \mathrm{H}_{2} \mathrm{O}, 9.53 \mathrm{~g} \mathrm{Na}_{2} \mathrm{HPO}_{4}$, $4.05 \mathrm{~g} \mathrm{KH}_{2} \mathrm{PO}_{4}, 1 \mathrm{~g}$ glucose, $15 \mathrm{mg} \mathrm{CaCl}_{2} \cdot 2 \mathrm{H}_{2} \mathrm{O}, 1 \mathrm{~mL}$ trace metal solution, $0.5 \mathrm{mmol}$ Trp, and $1 \mathrm{mmol} P P Y$. Feed solution contained per liter: $600 \mathrm{~g}$ glucose, $6 \mathrm{~g} \mathrm{MgSO}_{4} \cdot 7 \mathrm{H}_{2} \mathrm{O}, 150 \mathrm{mg}$ $\mathrm{CaCl}_{2} \cdot 2 \mathrm{H}_{2} \mathrm{O}, 10 \mathrm{mmol}$ Trp, $100 \mathrm{mg}$ kanamycin, and $10 \mathrm{~mL}$ trace metal solution. Phenylpyruvate was fed by syringe based on $0.307 \mathrm{mmol}$ PPY $\mathrm{g}_{\mathrm{CDW}}{ }^{-1}$. The PPY demand was calculated based on the abundance of Phe and Tyr in the E. coli biomass composition (Pramanik and Keasling, 1997), since both bacteria belongs to Gammaproteobacteria.

\section{Cultivation Conditions}

All shake flask incubation was carried out in baffled Erlenmeyer flasks in an incubator $(2.5 \mathrm{~cm}$ orbit, Multitron, Infors, Bottmingen, Switzerland) at $200 \mathrm{rpm}$. Incubation temperature for flasks and plates was $37^{\circ} \mathrm{C}$ for E. coli and $30^{\circ} \mathrm{C}$ for P. putida.

To select best performing strain, the PHBA production strains were first cultivated for $8 \mathrm{~h}$ in $5 \mathrm{~mL} \mathrm{LB}$. Fifty microliters of this culture was inoculated into a fresh $25-\mathrm{mL}$ CDM medium overnight, and the next day was inoculated to $35-\mathrm{mL} \mathrm{CDM}$ medium in a $250-\mathrm{mL}$ baffled flask to the initial $\mathrm{OD}_{600}$ of 0.2 . The inducer isopropyl $\beta$-D-1-thiogalactopyranoside (IPTG) was added to a final concentration of $1 \mathrm{mmol} \mathrm{L}^{-1}$ when the $\mathrm{OD}_{600}$ reached 0.3-0.4. Two additional feeds to the final concentration of $0.5 \mathrm{mM}$ PPY and $5 \mathrm{~g} \mathrm{~L}^{-1}$ glucose were added at 6 and $20 \mathrm{~h}$, respectively. Samples were centrifuged $(16,000 \mathrm{~g}, 10 \mathrm{~min}$, room temperature) and supernatants were stored in the freezer $\left(-20^{\circ} \mathrm{C}\right)$ for further analysis.

Fed-batch fermentations with $P$. putida were carried out in two 1.0 L working volume Biostat $\mathrm{B}+$ bioreactors (Sartorius, Germany), with initial volumes of $0.4 \mathrm{~L}$. Temperature was set at $30 \pm 0.5^{\circ} \mathrm{C}$ and $\mathrm{pH}$ was automatically controlled at $7.0 \pm 0.1 \mathrm{using}$ $14-15 \%(\mathrm{w} / \mathrm{v}) \mathrm{NH}_{4} \mathrm{OH}$, which also served as additional nitrogen source for cell growth. Dissolved oxygen (DO) was maintained at a minimum of $40 \%$ through automatic control of the agitation between 500 and $2000 \mathrm{rpm}$ followed by variation of air flow from 0.15 to $1.5 \mathrm{~L} \mathrm{~min}^{-1}$. Seed cultures were grown in seed medium at $30^{\circ} \mathrm{C}, 200 \mathrm{rpm}$ flasks overnight (around 13-16 h), collected in mid-exponential phase and then inoculated into the fermenter with initial $\mathrm{OD}_{600}$ of $1.0 \pm 0.1$. The inducer IPTG was added in the early exponential phase (3-4 h), with an initial concentration of $2.5 \mathrm{mM}$. The feed phase was started half an hour after inoculation (Figure S3 in Supplementary Material). The antifoam polypropylene glycol P2000 (Sigma-Aldrich, Australia) was added when onset of foaming was observed.

\section{DNA Manipulation}

Gene Deletions ( $\Delta$ pobA, $\Delta p h e A, \Delta \operatorname{trpE}$, and $\Delta h e x R$ ) Gene deletions were performed in $P$. putida following a multiple, seamless, and marker-less genomic editing method from Martinez-Garcia and de Lorenzo (2011). The resulting strains are listed in Table 1, and gene knockout process or overexpression is shown in Figure S1 in Supplementary Material. Primers used in this study are listed in Table S2 in Supplementary Material. The pEMG-based knockout plasmids were constructed using a 
standard restriction and ligation approach. To ease the constructions, the nested fusion PCR approach was applied to increase PCR amplification specificity (Figure S1A in Supplementary Material). Briefly, the fusion gene fragments and $\mathrm{pEMG}$ vector were digested with $B a m \mathrm{HI} / E c o$ RI, ligated with a T4 DNA ligase, and transformed into chemical competent cells (E. coli DH5 $\alpha$ $\lambda$ pir). The positive transformants were obtained from blue-white screening, and further verified with colony PCR, restriction digestion, and sequencing (performed by Australian Genome Research Facility Ltd.), resulting in the four knockout plasmids pEMG- $\Delta$ pobA, pEMG- $\Delta$ pheA, pEMG- $\Delta \operatorname{tr} p E$, and pEMG- $\Delta$ hexR. The plasmids were electro-transformed into $P$. putida-competent cells according to Choi's protocol (Choi and Schweizer, 2006). The resulting strains were transformed with the plasmid expressing ISce-I endonuclease (pSW-2) for cutting the pEMG vector backbone (Figure S1B in Supplementary Material). Then, the strains were induced with $15 \mathrm{mM} \mathrm{3-methylbenzoate} \mathrm{for} \mathrm{6-8} \mathrm{h,}$ and spread on LB plates containing $50 \mu \mathrm{g} \mathrm{mL} \mathrm{m}^{-1}$ gentamycin. Finally, selected mutant strains were confirmed using colony PCR with primers of P2/P5. The desired mutant should have the expected size of around 1000 bp (Table S2 in Supplementary Material). Auxotrophic mutants were also verified on solid CDM medium plate, $5 \mathrm{~g} \mathrm{~L}^{-1}$ glucose with and without $0.5 \mathrm{mM}$ Trp, and/or $0.5 \mathrm{mM}$ Phe and $0.5 \mathrm{mM}$ Tyr supplementation (the deletion of gene $\operatorname{trp} E$ generated the tryptophan auxotroph, and the deletion of gene pheA yielded tyrosine and phenylalanine double auxotrophic mutants).

\section{Expression Plasmid Construction}

The genes $u b i C$ encoding chorismate lyase from E. coli $\mathrm{K}-12$ substr. W3110 (CAA40681.1) and aro $G^{D 146 N}$ encoding feedback insensitive DAHP synthase (Kikuchi et al., 1997) were codon optimized and synthesized by Integrated DNA Technologies, Inc. These two genes were digested with EcoRI, SalI, and HindIII, respectively, then inserted into the vector pSEVA234 (SilvaRocha et al., 2013), resulting in the plasmids pSEVA234-ubiC and pSEVA234-UA (Figure S2C in Supplementary Material). The sequencing-verified plasmids were electro-transformed into each of the knockout mutant $P$. putida strains.

\section{Sodium Dodecyl Sulfate Polyacrylamide Gel Electrophoresis}

Culture samples for sodium dodecyl sulfate polyacrylamide gel electrophoresis (SDS-PAGE) were collected by centrifugation $(16,000 \mathrm{~g}, 5 \mathrm{~min}$, and room temperature). Pellets were washed with buffer once and resuspended with $50 \mathrm{mM}$ Tris- $\mathrm{HCl}$ buffer $(\mathrm{pH}$ 7.5). Cells were then disrupted using sonication for 5-10 cycles of $10 \mathrm{~s}$ pulse with $10 \mathrm{~s}$ interval on ice to prepare protein extracts. For preparation of whole-cell protein samples, the loading buffer was added directly to the crude lysate. For the analysis of soluble protein expression, the cell lysate was first centrifuged at $16,000 \mathrm{~g}$ for $20 \mathrm{~min}$ at $4^{\circ} \mathrm{C}$ to remove cell debris and insoluble particles. Then, the loading buffer containing $\beta$-mercaptoethanol and SDS was added, and heated $\left(5 \mathrm{~min}, 95-99^{\circ} \mathrm{C}\right)$ to denature the protein. SDS-PAGE was performed using $12 \%$ polyacrylamide gel according to Laemmli's protocol (Laemmli, 1970) in a vertical slab gel apparatus from Bio-Rad Laboratories. Coomassie brilliant blue G-250 was used for gel staining.

\section{Analytical Methods}

Pseudomonas putida cell densities were monitored by a spectrophotometry (GENESYS 10S UV-Vis, USA) at the wavelength of $600 \mathrm{~nm}\left(\mathrm{OD}_{600}\right)$ against water. The correlation between $\mathrm{OD}$ and cell dry weight (CDW) was empirically determined to be CDW $\left(\mathrm{g} \mathrm{L}^{-1}\right)=0.486 \mathrm{OD}_{600}$. Sugars, organic acids (Lai et al., 2016a), and aromatic compounds (Lai et al., 2016b) were measured by high-performance liquid chromatography (HPLC). Carbon dioxide was monitored by gas analyzer (Balzers Thermostar GSD 300 T3, Liechtenstein). Carbon balance calculation was following the equation of $\mathrm{F}(\mathrm{C}$, in $)=\mathrm{F}(\mathrm{C}$, out $) \approx \mathrm{F}(\mathrm{C}$, sugars $)+\mathrm{F}(\mathrm{C}$, organic acids $)+\mathrm{F}(\mathrm{C}$, aromatics $)+\mathrm{F}\left(\mathrm{C}, \mathrm{CO}_{2}\right)+\mathrm{F}(\mathrm{C}$, biomass $)$. Specifically, carbon composition in $P$. putida KT2440 biomass is 48.8\% ( $\mathrm{W} \mathrm{W}^{-1}$ ) (van Duuren et al., 2013).

\section{RESULTS AND DISCUSSION}

\section{Metabolic Engineering of PHBA Production Strains}

There is a solid body of research for the metabolic engineering of shikimate pathway-derived aromatics, mainly using E. coli and Corynebacterium glutamicum. P. putida strain development for the production of PHBA can be built on this knowledge and apply successful genetic engineering strategies. Knockout targets were chosen based on their ability to degrade PHBA or to compete with the enzyme responsible for PHBA production (UbiC) for precursor availability. Compared to E. coli and C. glutamicum, more unique to $P$. putida strains is the ability for aromatics degradation and the multitude of degradation pathways for different aromatics (Jiménez et al., 2002). To increase PHBA concentration, the genes $p o b A$, pheA, $\operatorname{trp} E$, and hexR were deleted from the genome of $P$. putida KT2440 using the pEMG/pSW-2 system (Martinez-Garcia and de Lorenzo, 2011). The pobA gene encodes p-hydroxy benzoate hydroxylase and causes product degradation. The gene pheA encodes the bifunctional enzyme of prephenate dehydratase and chorismate mutase, this will direct carbon flux into the tyrosine and phenylalanine synthesis, and is a major competitor for the precursor chorismate. The disruption of $\operatorname{trp} E$, which encodes anthranilate synthase component I, is likely to reduce the drain of chorismate into the tryptophan biosynthesis. As a consequence, the deletion of $p h e A$ and $\operatorname{tr} p E$ was carried out to increase precursor supply to PHBA production by blocking the branching of the flux toward the synthesis of the aromatic amino acids (Figure 2). Both deletions resulted in aromatic amino acids auxotrophic mutants, but when depleted would cause the natural feedback inhibition of the shikimate pathway to be alleviated. It is also important to note that Pseudomonas species can degrade Phe and Tyr through the homogentisate pathway, which can cause a difficulty to screen $\Delta$ phe mutant and the inconvenience in fedbatch fermentation. Phe is first converted into Tyr by the action of the gene products of phhAB phenylalanine hydroxylase, which are regulated by the $\mathrm{PhhR}$ regulator protein in the presence of the two amino acids (Herrera and Ramos, 2007). They are then 


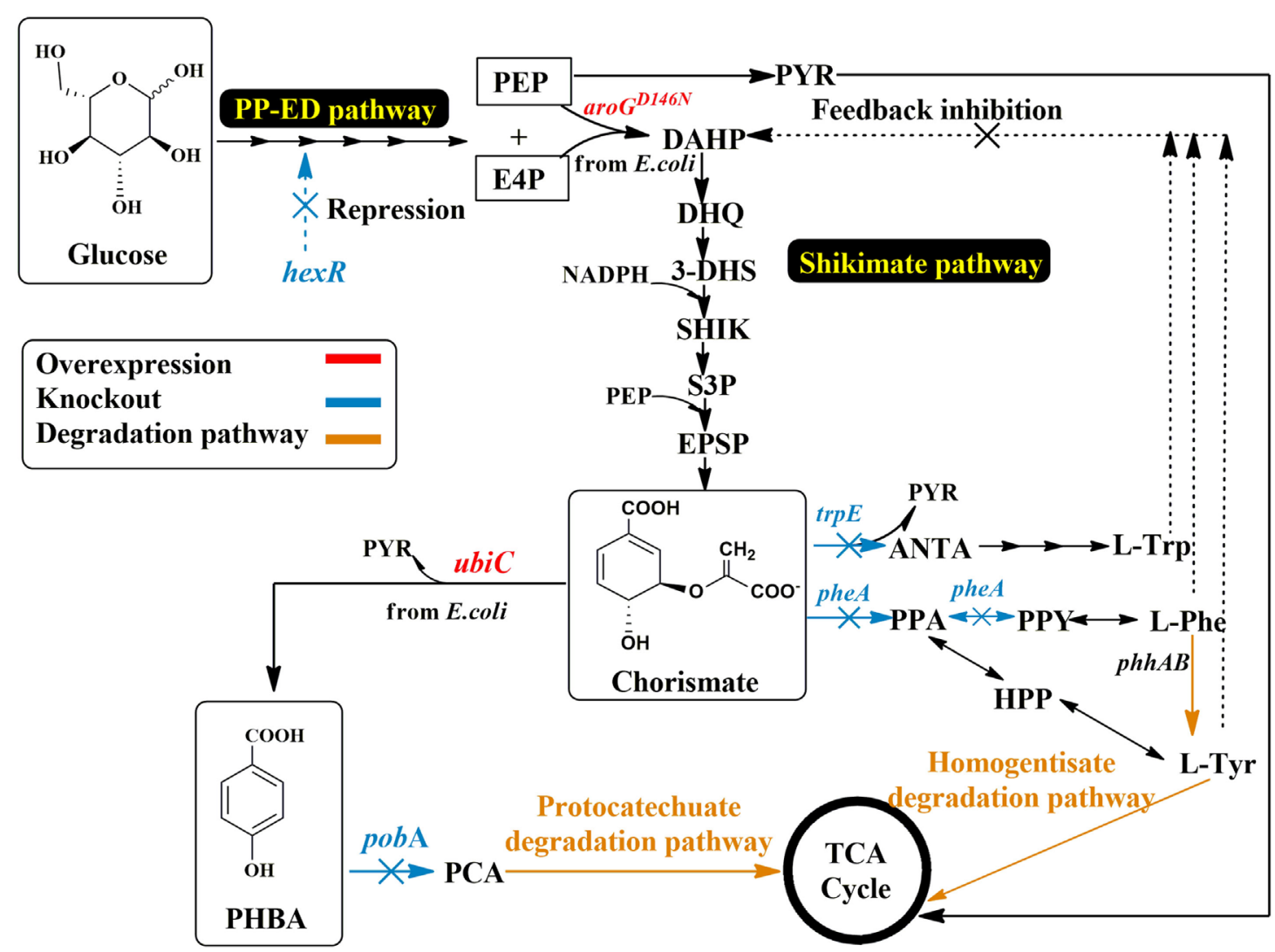

FIGURE 2 | Schematic view of metabolic engineering in $\boldsymbol{P}$ putida for PHBA production. AroG ${ }^{\mathrm{D} 146 \mathrm{~N}}$, feedback resistant DAHP synthase; E4P, erythrose 4-phosphate; HPP, 4-hydroxyphenyl pyruvate; PEP, phosphoenolpyruvate; ANAT, anthranilate; PheA, chorismate mutase/prephenate dehydratase; PhhAB, phenylalanine hydroxylase; PPA, prephenate; PPY, phenylpyruvate; PobA, p-hydroxy benzoate hydroxylase; TrpE, anthranilate synthase component I; UbiC, chorismate lyase; HexR, repressor for glucose metabolism.

further degraded via the homogentisate degradation pathway, and metabolites are channeled to the tricarboxylic acid (TCA) cycle (Arias-Barrau et al., 2004). Glucose metabolism repressor hexR controls glucokinase $(g l u)$, glyceraldehyde-3-phosphate (gap-1), and several Entner-Doudoroff pathway enzymes ( $z w f$, $p g l$, edd, and eda), the deletion of this repressor may increase the flux toward pentose phosphate pathway (PPP) and NADPH pool. The deletion of hexR was expected to increase availability of shikimate pathway precursors $\mathrm{E} 4 \mathrm{P}$ and the necessary reducing equivalents NADPH (Figure 2). Successful deletants were chosen based on LB screening with the appropriate antibiotic selection as described in Section "Materials and Methods."

Chorismate lyase encoded by $u b i C$ is a key enzyme in PHBA synthesis; it specifically hydrolyzes chorismate into the products PHBA and pyruvate. The $u b i C$ gene from E. coli K-12 strain was cloned into vector pSEVA234 regulated with $\mathrm{Ptrc} / \mathrm{LacI}^{\mathrm{q}}$. E. coli wild-type UbiC enzyme was shown to be insoluble when overexpressed in E. coli, protein manipulations made to increase solubility resulted in some loss of enzyme activity (Stover et al., 2000; Holden et al., 2002). To optimize expression of soluble UbiC, different culture temperature $\left(25\right.$ and $\left.30^{\circ} \mathrm{C}\right)$ and induction time ( 6 and $8 \mathrm{~h}$ ) were performed. However, unlike previous work in E. coli, we did not observe protein precipitation in P. putida in any of the tested conditions. Instead, over $90 \%$ of $E$. coli wild type UbiC was present in soluble form in P. putida KT2440 using culture condition as described above (Figure S2 and Table S1 in Supplementary Material).

Finally, to further improve the production of PHBA in P. putida, the gene encoding the feedback insensitive 3-deoxy-D-arabinoheptulosonate 7-phosphate (DAHP) synthase isoenzyme mutant AroG $^{\text {D146N }}$ (Kikuchi et al., 1997) was constructed in the same operon with UbiC, regulated by the $\mathrm{Ptrc} / \mathrm{LacI}^{\mathrm{q}}$ system (Figure $\mathrm{S} 1 \mathrm{C}$ in Supplementary Material). This caused an unregulated influx of carbon into the shikimate pathway.

On a side note, it was earlier postulated by Kuepper et al. (2015) after screening more than a thousand colonies for pheA knockout mutants on complex medium that the presence of the homogentisate degradation pathway might hamper the positive identification of positive colonies and the addition of precursor PPY in minimal medium was helpful to screen for this mutant (Kuepper et al., 2015). We successfully identified pheA mutants on rich medium when looking for tiny colonies that could be easily overlooked amongst the big colonies that did not contain the knockout. 


\section{Evaluation of the PHBA Production Strains in Shake Flasks}

The performance of the genetically engineered strains described above (S1-S6, Table 1) was evaluated for PHBA production in shake flasks, showing a maximum yield of $1.30 \pm 0.01 \mathrm{mmol}_{\text {PHBA }} \mathrm{g}_{\mathrm{CDW}}{ }^{-1}$, a titer of $4.96 \pm 0.146 \mathrm{mM}$, and 7\% (C-mol C-mol ${ }^{-1}$ ) (Figure 3). The single knockout strain containing the over expression plasmid of $u b i C$ (strain S1) produced $0.23 \mathrm{mM}$ PHBA with a yield of $0.075 \mathrm{mmol}_{\mathrm{PHBA}} \mathrm{g}_{\mathrm{CDW}}{ }^{-1}$. An increase of around fivefold in titer and yield was achieved with the deletion of chorismate precursor competing genes pheA (strain S2). Compared to S1, this was further increased to 12- and 16-fold increase in titer and yield, respectively, by the deletion of the second precursor competing gene $\operatorname{trpE}$ (strain S3). Overall, indicating a positive effect when deleting precursor competing genes. The knockout of hexR in the S3 background did not lead to a very large increase in yield and titer (strain S4) nor did the overexpression of the feedback resistant DAHP synthase (strain S5). However, when combining the knockout of hexR with overexpression of DAHP synthase (strain S6) a remarkable increase of 22- and 17-fold increase in titer and yield was achieved relative to the base strain. Compared to S3, S4 has no significant increase in yield and titer, and it can be explained by the fact that our limited feeding of phenylpruvate and tryptophan alleviated or diminished feedback inhibition to their native DAHP synthase in $P$. putida. Unexpectedly, compared to S3, the hexR knockout has no substantial improvement for PHBA production but the hexR knockout strain combined overexpression of DAHP synthase, has a significant improvement in titer and yield. The possible reason that the native DAHP synthase was not strong enough to drain flux from central metabolism, interestingly, the combination of both genetic manipulations can synergistically

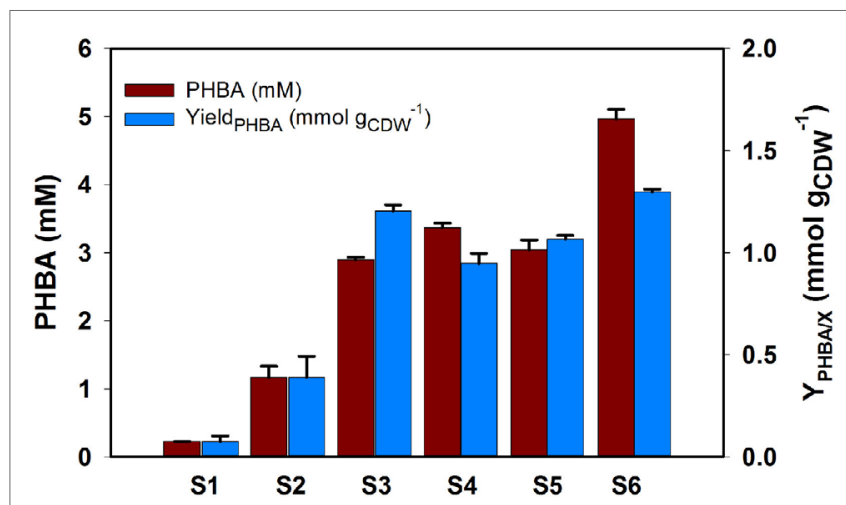

FIGURE 3 | Various $P$. putida KT2440 mutant strains for PHBA production in shake flask. All mutants were grown in CDM medium supplemented with $5 \mathrm{~g} \mathrm{~L}^{-1}$ glucose and phenyl pyruvate and tryptophan as described above. Two additional feeds of $5 \mathrm{~g} \mathrm{~L}^{-1}$ glucose and $0.5 \mathrm{mM}$ PPY were added after 6 and $20 \mathrm{~h}$, respectively. All data were from triplicate biological repeats at the late stationary time point of $44 \mathrm{~h}$. Genotypes for mutants tested in this study. S1, KT $\Delta$ pobA/234-ubiC; S2,

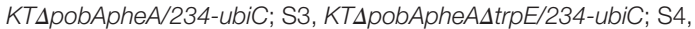

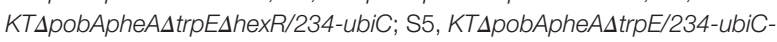

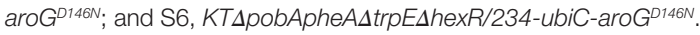

drain the flux from central metabolism to shikimate pathway for the PHBA production, leading to a significant improvement either in titer or in yield. The reason for the increase could be that Pseudomonas, like many bacteria, features an imbalanced supply of PEP and erythrose 4-phosphate (E4P), and increasing PPP flux was demonstrated to increase aromatic production in several bacteria including $P$. putida (Chassagnole et al., 2002; Fuhrer et al., 2005; Nikel et al., 2015). The general strategies to increase the E4P supply are to express genes from PP pathway such as tktA encoding transketolase and/or tal encoding transaldolase (Lu and Liao, 1997; Ikeda and Katsumata, 1999). The role of the hexR protein as a transcriptional repressor of $z w f$ encoding the committing step into PPP was described in P. putida and its deletion led to increased flux of PPP and reduction of the $\mathrm{NADP}^{+}$pool (del Castillo et al., 2008; Meijnen et al., 2012). In our study, titer increased significantly in the S6 strain, likely due to an increased precursor supply for our PHBA production from glucose.

\section{PHBA Production by Fed-Batch Fermentation}

The best PHBA producing strain (S6) was selected from the flask experiment to evaluate its potential in a fed-batch fermentation. P. putida will quickly degrade the provided aromatic amino acids Phe and Tyr in fermentation due to the homogentisate degradation pathway. This would lead to growth arrest in our $\Delta p h e A$ strains, or the need for a constant feed of those amino acids. Instead of feeding the two amino acids, we fed the precursor PPY. This feed did not stop degradation through the homogentisate pathway completely but slowed down the process. We assumed P. putida KT2440 has a similar biomass composition to E. coli, since both species are $\gamma$-proteobacteria (Blank et al., 2008) and calculated the necessary PPY feed based on the demand for Tyr and Phe. Glucose concentration was $1 \mathrm{~g} \mathrm{~L}^{-1}$ at the start of the fermentation and was fed as described above after inocula-

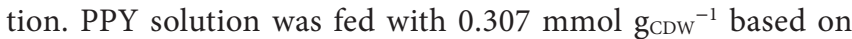
the actual cell density at several intervals $(5,8,10,15,20$, and $25 \mathrm{~h}$ ). Biomass kept growing after $\mathrm{OD}_{600}$ reached $\sim 12$ and $6.05 \mathrm{~g}$ CDW were obtained after $32 \mathrm{~h}$. Fermentation was stopped when the working volume of the reactor was reached. Due to the volume change in the fed-batch the following section will discuss absolute amounts. The carbon balance closed at $100.6 \%$ meaning that all carbon could be accounted for. As the major by-products, $\alpha$-keto-gluconate was accumulated $(545 \mathrm{mmol})$ and $\mathrm{CO}_{2}$ was released $(177 \mathrm{mmol})$ and about half the fed glucose ( 765 mmol) remained unused. This was due to the fact that glucose was not limiting in the fed-batch but PPY instead. $P$. putida will oxidize glucose in periplasm to gluconate and then to $\alpha$-keto-gluconate. In future experiments, the feed regime should be optimized. Nevertheless, PHBA accumulated over time and reached a maximum titer of $12.46 \mathrm{mmol}(12.5 \mathrm{mM}$ at

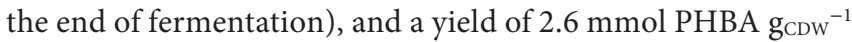
(Figure 4). The product on substrate yield of PHBA based on the consumed $\mathrm{C}_{6}$ molecules and PPY (excluding extracellular glucose and $\alpha$-keto-gluconate, which were not taken up by the cells), reached $18.1 \%\left(\mathrm{C}-\mathrm{mol} \mathrm{C}-\mathrm{mol}^{-1}\right)$. Smaller amounts 


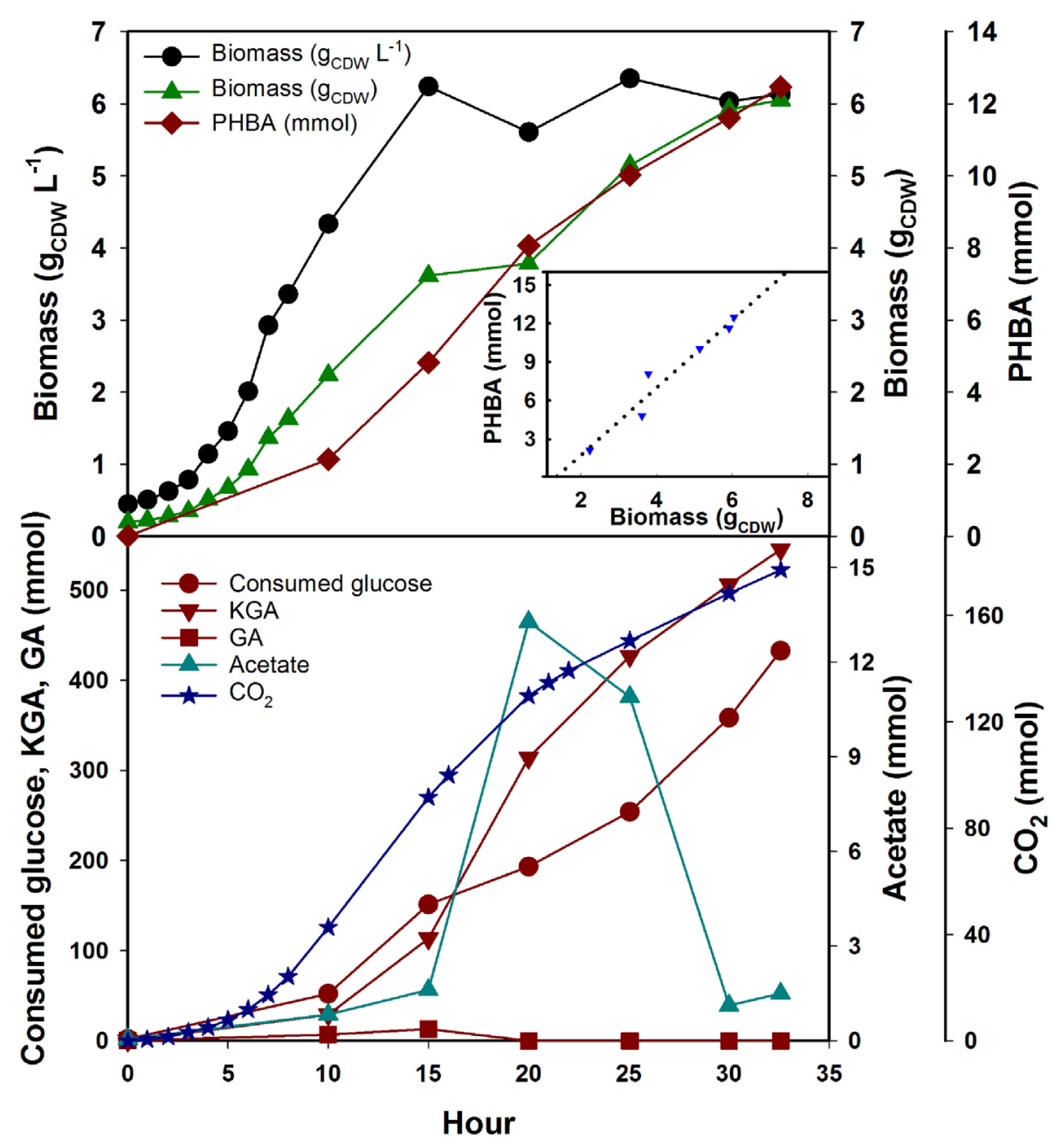

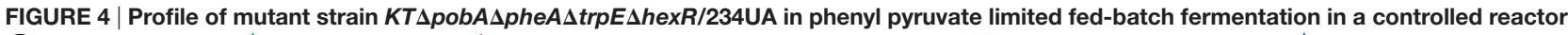

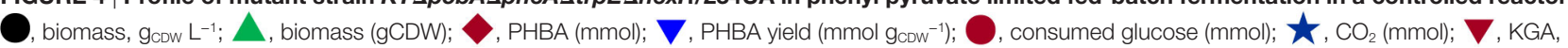

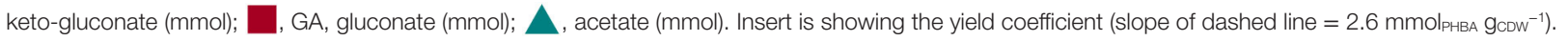

of pyruvate, lactate, and succinate could also be observed to accumulate throughout the fermentation (data not shown). The only other major by-product was acetate at $13 \mathrm{mmol}$ which accumulated in the first $20 \mathrm{~h}$ and was consumed thereafter (Figure 4). This might indicate a redirection of intracellular fluxes around the 20 -h mark, but this remains to be studied in more detail.

In summary, the fed-batch process was able to achieve higher carbon yields than the shake flask experiments, but the feeding still requires optimization. Compared to published results we

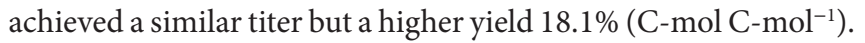
P. putida S12palB1 (8.5 C-mol C-mol ${ }^{-1}$ ) was engineered based on the coumarate pathway and had optimized carbon flux toward L-tyrosine (Verhoef et al., 2007), and P. putida S12pal_xylB7 (16.3 C-mol C- $\mathrm{mol}^{-1}$ ) used an optimized mixed-substrate feeding strategy and also the coumarate pathway (Meijnen et al., 2011). While the experimentally observed yields are not yet approaching the calculated theoretical yields of the pathways (Krömer et al., 2013), this might still indicate that the UbiC based route has a higher potential for biotechnological production of PHBA.

\section{CONCLUSION}

Pseudomonas putida strains show high potential for industrial aromatic production, mainly due to their high tolerance toward toxic aromatics and the reduced formation of by-products. In this paper, we described P. putida production of PHBA from glucose as sole carbon source $v i a$ the chorismate lyase from $E$. coli, which has a higher theoretical yield than the previously described coumarate

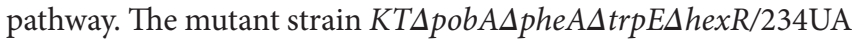
achieved a maximum titer of $12.5 \mathrm{mM}$ in a PPY limited fedbatch fermentation. This was achieved using a stable, scar-less, and marker-less deletion method, to meet industrial application requirements. This study lays the basis for using $P$. putida as a 
PHBA production host. Further metabolic engineering strategies can be applied to enhance production, for example, balancing the precursors PEP and E4P or increasing the availability of the reducing equivalent NADPH.

\section{AUTHOR CONTRIBUTIONS}

SY performed the experiments, designed the study, analyzed data, and drafted the manuscript. MP performed quantitative analytics. GW co-supervised the study and edited the manuscript. JK designed and supervised the study, analyzed data, and edited the manuscript. MP performed HPLC operation, and JO and SY performed carbon balance analysis.

\section{REFERENCES}

Arias-Barrau, E., Olivera, E. R., Luengo, J. M., Fernández, C., Galán, B., García, J. L., et al. (2004). The homogentisate pathway: a central catabolic pathway involved in the degradation of L-phenylalanine, L-tyrosine, and 3-hydroxyphenylacetate in Pseudomonas putida. J. Bacteriol. 186, 5062-5077. doi:10.1128/ jb.186.15.5062-5077.2004

Averesch, N. J. H., Winter, G., and Krömer, J. O. (2016). Production of para-aminobenzoic acid from different carbon-sources in engineered Saccharomyces cerevisiae. Microb. Cell Fact. 15, 1-16. doi:10.1186/ s12934-016-0485-8

Barker, J. L., and Frost, J. W. (2001). Microbial synthesis of $p$-hydroxybenzoic acid from glucose. Biotechnol. Bioeng. 76, 376-390. doi:10.1002/Bit.10160

Bentley, R. (1990). The shikimate pathway - a metabolic tree with many branches. Crit. Rev. Biochem. Mol. Biol. 25, 307-384. doi:10.3109/10409239009090615

Blank, L., Ionidis, G., Ebert, B., Buhler, B., and Schmid, A. (2008). Metabolic response of Pseudomonas putida during redox biocatalysis in the presence of a second octanol phase. FEBS J. 275, 5173-5190. doi:10.1111/j.1742-4658.2008.06648.x

Chassagnole, C., Noisommit-Rizzi, N., Schmid, J. W., Mauch, K., and Reuss, M. (2002). Dynamic modeling of the central carbon metabolism of Escherichia coli. Biotechnol. Bioeng. 79, 53-73. doi:10.1002/bit.10288

Chavarría, M., Nikel, P. I., Pérez-Pantoja, D., and de Lorenzo, V. (2013). The Entner-Doudoroff pathway empowers Pseudomonas putida KT2440 with a high tolerance to oxidative stress. Environ. Microbiol. 15, 1772-1785. doi:10.1111/1462-2920.12069

Choi, K. H., and Schweizer, H. P. (2006). Mini-Tn7 insertion in bacteria with single att $\operatorname{Tn} 7$ sites: example Pseudomonas aeruginosa. Nat. Protoc. 1, 153-161. doi:10.1038/nprot.2006.24

Curran, K. A., Leavitt, J. M., Karim, A. S., and Alper, H. S. (2013). Metabolic engineering of muconic acid production in Saccharomyces cerevisiae. Metab. Eng. 15, 55-66. doi:10.1016/j.ymben.2012.10.003

del Castillo, T., Duque, E., and Ramos, J. L. (2008). A set of activators and repressors control peripheral glucose pathways in Pseudomonas putida to yield a common central intermediate. J. Bacteriol. 190, 2331-2339. doi:10.1128/jb.01726-07

Ebert, B. E., Kurth, F., Grund, M., Blank, L. M., and Schmid, A. (2011). Response of Pseudomonas putida KT2440 to increased NADH and ATP demand. Appl. Environ. Microbiol. 77, 6597-6605. doi:10.1128/aem.05588-11

Erickson, B., Nelson, J. E., and Winters, P. (2012). Perspective on opportunities in industrial biotechnology in renewable chemicals. Biotechnol. J. 7, 176-185. doi:10.1002/biot.201100069

Fuhrer, T., Fischer, E., and Sauer, U. (2005). Experimental identification and quantification of glucose metabolism in seven bacterial species. J. Bacteriol. 187, 1581-1590. doi:10.1128/jb.187.5.1581-1590.2005

Gavrilescu, M. (2014). "Biomass potential for sustainable environment, biorefinery products and energy," in Sustainable Energy in the Built Environment - Steps Towards nZEB: Proceedings of the Conference for Sustainable Energy (CSE) 2014, ed. I.Visa (Cham: Springer International Publishing), 169-194.

Gosset, G. (2009). Production of aromatic compounds in bacteria. Curr. Opin. Biotechnol. 20, 651-658. doi:10.1016/j.copbio.2009.09.012

Herrera, M. C., and Ramos, J.-L. (2007). Catabolism of phenylalanine by Pseudomonas putida: the NtrC-family PhhR regulator binds to two sites

\section{ACKNOWLEDGMENTS}

The authors thank the University of Queensland Graduate School for scholarship support of SY. JK was partially supported by the Australian Research Council (DE120101549). They would like to also thank Bin Lai and Endah Agustina Lestari for the help of fermentation.

\section{SUPPLEMENTARY MATERIAL}

The Supplementary Material for this article can be found online at http://journal.frontiersin.org/article/10.3389/fbioe.2016.00090/ full\#supplementary-material.

upstream from the phhA gene and stimulates transcription with $\sigma 70$. J. Mol. Biol. 366, 1374-1386. doi:10.1016/j.jmb.2006.12.008

Holden, M. J., Mayhew, M. P., Gallagher, D. T., and Vilker, V. L. (2002). Chorismate lyase: kinetics and engineering for stability. Biochim. Biophys. Acta 1594, 160-167. doi:10.1016/s0167-4838(01)00302-8

Ibeh, C. C. (2011). Thermoplastic Materials: Properties, Manufacturing Methods, and Applications. Boca Raton, FL: CRC Press.

Ikeda, M., and Katsumata, R. (1999). Hyperproduction of tryptophan by Corynebacterium glutamicum with the modified pentose phosphate pathway. Appl. Environ. Microbiol. 65, 2497-2502.

Jiménez, J. I., Miñambres, B., García, J. L., and Díaz, E. (2002). Genomic analysis of the aromatic catabolic pathways from Pseudomonas putida KT2440. Environ. Microbiol. 4, 824-841. doi:10.1046/j.1462-2920.2002.00370.x

Karpf, M., and Trussardi, R. (2009). Efficient access to oseltamivir phosphate (Tamiflu) via the O-trimesylate of shikimic acid ethyl ester. Angew. Chem. Int. Ed. Engl. 48, 5760-5762. doi:10.1002/anie.200901561

Kikuchi, Y., Tsujimoto, K., and Kurahashi, O. (1997). Mutational analysis of the feedback sites of phenylalanine-sensitive 3-deoxy-D-arabino-heptulosonate-7-phosphate synthase of Escherichia coli. Appl. Environ. Microbiol. 63, 761-762.

Koma, D., Yamanaka, H., Moriyoshi, K., Ohmoto, T., and Sakai, K. (2012). Production of aromatic compounds by metabolically engineered Escherichia coli with an expanded shikimate pathway. Appl. Environ. Microbiol. 78, 6203-6216. doi:10.1128/aem.01148-12

Krömer, J. O., Nunez-Bernal, D., Averesch, N. J. H., Hampe, J., Varela, J., and Varela, C. (2013). Production of aromatics in Saccharomyces cerevisiae - a feasibility study. J. Biotechnol. 163, 184-193. doi:10.1016/ j.jbiotec.2012.04.014

Kuepper, J., Dickler, J., Biggel, M., Behnken, S., Jaeger, G., Wierckx, N., et al. (2015). Metabolic engineering of Pseudomonas putida KT2440 to produce anthranilate from glucose. Front. Microbiol. 6:1310. doi:10.3389/ fmicb. 2015.01310

Laemmli, U. K. (1970). Cleavage of structural proteins during the assembly of the head of bacteriophage T4. Nature 227, 680-685. doi:10.1038/227680a0

Lai, B., Plan, M., Hodson, M., and Krömer, J. O. (2016a). Simultaneous determination of sugars, carboxylates, alcohols and aldehydes from fermentations by high performance liquid chromatography. Fermentation 2, 6. doi:10.3390/ fermentation 2010006

Lai, B., Plan, M. R., Averesch, N. J. H., Yu, S., Kracke, F., Lekieffre, N., et al. (2016b) Quantitative analysis of aromatics for synthetic biology using liquid chromatography. Biotechnol. J. doi:10.1002/biot.201600269

Le Meur, S., Zinn, M., Egli, T., Thony-Meyer, L., and Ren, Q. (2012). Production of medium-chain-length polyhydroxyalkanoates by sequential feeding of xylose and octanoic acid in engineered Pseudomonas putida KT2440. BMC Biotechnol. 12:53. doi:10.1186/1472-6750-12-53

Lennox, E. S. (1955). Transduction of linked genetic characters of the host by bacteriophage P1. Virology 1, 190-206. doi:10.1016/0042-6822(55) 90016-7

Lin, Y., Sun, X., Yuan, Q., and Yan, Y. (2014). Extending shikimate pathway for the production of muconic acid and its precursor salicylic acid in Escherichia coli. Metab. Eng. 23, 62-69. doi:10.1016/j.ymben.2014.02.009 
Lu, J.-L., and Liao, J. C. (1997). Metabolic engineering and control analysis for production of aromatics: role of transaldolase. Biotechnol. Bioeng. 53, 132-138. doi:10.1002/(SICI)1097-0290(19970120)53:2<132:AID-BIT2>3.0.CO;2-P

Martinez-Garcia, E., and de Lorenzo, V. (2011). Engineering multiple genomic deletions in Gram-negative bacteria: analysis of the multi-resistant antibiotic profile of Pseudomonas putida KT2440. Environ. Microbiol. 13, 2702-2716. doi:10.1111/j.1462-2920.2011.02538.x

Meijnen, J.-P., de Winde, J. H., and Ruijssenaars, H. J. (2012). Metabolic and regulatory rearrangements underlying efficient $\mathrm{D}$-xylose utilization in engineered Pseudomonas putida S12. J. Biol. Chem. 287, 14606-14614. doi:10.1074/jbc. M111.337501

Meijnen, J. P., Verhoef, S., Briedjlal, A. A., de Winde, J. H., and Ruijssenaars, H. J. (2011). Improved $p$-hydroxybenzoate production by engineered Pseudomonas putida S12 by using a mixed-substrate feeding strategy. Appl. Microbiol. Biotechnol. 90, 885-893. doi:10.1007/s00253-011-3089-6

Müller, R., Wagener, A., Schmidt, K., and Leistner, E. (1995). Microbial production of specifically ring C-13 labeled 4-hydroxybenzoic acid. Appl. Microbiol. Biotechnol. 43, 985-988. doi:10.1007/BF00166913

Ng, C. Y., Farasat, I., Maranas, C. D., and Salis, H. M. (2015). Rational design of a synthetic Entner-Doudoroff pathway for improved and controllable NADPH regeneration. Metab. Eng. 29, 86-96. doi:10.1016/ j.ymben.2015.03.001

Nikel, P. I., Chavarría, M., Fuhrer, T., Sauer, U., and de Lorenzo, V. (2015). Pseudomonas putida KT2440 strain metabolizes glucose through a cycle formed by enzymes of the Entner-Doudoroff, Embden-Meyerhof-Parnas, and pentose phosphate pathways. J. Biol. Chem. 290, 25920-25932. doi:10.1074/jbc. M115.687749

Poblete-Castro, I., Becker, J., Dohnt, K., dos Santos, V., and Wittmann, C. (2012). Industrial biotechnology of Pseudomonas putida and related species. Appl. Microbiol. Biotechnol. 93, 2279-2290. doi:10.1007/s00253-012-3928-0

Prakash, D., Pandey, J., Tiwary, B. N., and Jain, R. K. (2010). A process optimization for bio-catalytic production of substituted catechols 3-nitrocatechol and 3-methylcatechol. BMC Biotechnol. 10:49. doi:10.1186/1472-6750-10-49

Pramanik, J., and Keasling, J. D. (1997). Stoichiometric model of Escherichia coli metabolism: incorporation of growth-rate dependent biomass composition and mechanistic energy requirements. Biotechnol. Bioeng. 56, 398-421. doi:10.1002/ (SICI) 1097-0290(19971120)56:4<398:AID-BIT6>3.0.CO;2-J

Silva-Rocha, R., Martinez-Garcia, E., Calles, B., Chavarria, M., Arce-Rodriguez, A., de las Heras, A., et al. (2013). The Standard European Vector Architecture (SEVA): a coherent platform for the analysis and deployment of complex prokaryotic phenotypes. Nucleic Acids Res. 41, D666-D675. doi:10.1093/Nar/ Gks1119

Stover, C., Mayhew, M. P., Holden, M. J., Howard, A., and Gallagher, D. T. (2000). Crystallization and 1.1-Å diffraction of chorismate lyase from Escherichia coli. J. Struct. Biol. 129, 96-99. doi:10.1006/jsbi.1999.4205

Sun, Z., Ramsay, J., Guay, M., and Ramsay, B. (2006). Automated feeding strategies for high-cell-density fed-batch cultivation of Pseudomonas putida
KT2440. Appl. Microbiol. Biotechnol. 71, 423-431. doi:10.1007/s00253-005$0191-7$

Ütkür, F. Ö, Gaykawad, S., Bühler, B., and Schmid, A. (2011). Regioselective aromatic hydroxylation of quinaldine by water using quinaldine 4 -oxidase in recombinant Pseudomonas putida. J. Ind. Microbiol. Biotechnol. 38, 1067-1077. doi:10.1007/s10295-010-0883-6

van Duuren, J. B., Puchałka, J., Mars, A. E., Bücker, R., Eggink, G., Wittmann, C., et al. (2013). Reconciling in vivo and in silico key biological parameters of Pseudomonas putida KT2440 during growth on glucose under carbon-limited condition. BMC Biotechnol. 13:93. doi:10.1186/1472-6750-13-93

Verhoef, S., Ruijssenaars, H.J., deBont, J. A.M., and Wery, J. (2007). Bioproduction of $p$-hydroxybenzoate from renewable feedstock by solvent-tolerant Pseudomonas putida S12. J. Biotechnol. 132, 49-56. doi:10.1016/j.jbiotec.2007.08.031

Verhoef, S., Wierckx, N., Westerhof, R. G. M., de Winde, J. H., and Ruijssenaars, H. J. (2009). Bioproduction of $p$-hydroxystyrene from glucose by the solvent-tolerant bacterium Pseudomonas putida S12 in a two-phase water-decanol fermentation. Appl. Environ. Microbiol. 75, 931-936. doi:10.1128/aem.02186-08

Weber, C., Brueckner, C., Weinreb, S., Lehr, C., Essl, C., and Boles, E. (2012) Biosynthesis of cis,cis-muconic acid and its aromatic precursors, catechol and protocatechuic acid, from renewable feedstocks by Saccharomyces cerevisiae. Appl. Environ. Microbiol. 78, 8421-8430. doi:10.1128/aem.01983-12

Wierckx, N. J. P., Ballerstedt, H., de Bont, J. A. M., and Wery, J.(2005). Engineering of solvent-tolerant Pseudomonas putida S12 for bioproduction of phenol from glucose. Appl. Environ. Microbiol. 71, 8221-8227. doi:10.1128/ Aem.71.12.8221-8227.2005

Williams, T. C., Averesch, N. J. H., Winter, G., Plan, M. R., Vickers, C. E., Nielsen, L. K., et al. (2015). Quorum-sensing linked RNA interference for dynamic metabolic pathway control in Saccharomyces cerevisiae. Metab. Eng. 29, 124-134. doi:10.1016/j.ymben.2015.03.008

Yoshida, T., and Nagasawa, T. (2007). "Biological Kolbe-Schmitt carboxylation: possible use of enzymes for the direct carboxylation of organic substrates A2," in Future Directions in Biocatalysis, ed. T.Matsuda (Amsterdam: Elsevier Science B.V.), 83-105.

Zhang, H., and Stephanopoulos, G. (2013). Engineering E. coli for caffeic acid biosynthesis from renewable sugars. Appl. Microbiol. Biotechnol. 97, 3333-3341. doi:10.1007/s00253-012-4544-8

Conflict of Interest Statement: The authors declare that the research was conducted in the absence of any commercial or financial relationships that could be construed as a potential conflict of interest.

Copyright $\odot 2016 \mathrm{Yu}$, Plan, Winter and Krömer. This is an open-access article distributed under the terms of the Creative Commons Attribution License (CC BY). The use, distribution or reproduction in other forums is permitted, provided the original author(s) or licensor are credited and that the original publication in this journal is cited, in accordance with accepted academic practice. No use, distribution or reproduction is permitted which does not comply with these terms. 